\begin{tabular}{|c|l|}
\hline Title & Emission of terahertz radiation from two-dimensional electron systems in semiconductor nano-heterostructures \\
\hline Author(s) & $\begin{array}{l}\text { Otsuji, Taiichi; Karasawa, Hiromi; Watanabe, Takay uki; Suemitsu, Tetsuy a; Suemitsu, Maki; Sano, Eiich;; Knap, } \\
\text { Wojciech; Ryzhii, Victor }\end{array}$ \\
\hline Citation & $\begin{array}{l}\text { Comptes Rendus Physique, 11(7-8), 421-432 } \\
\text { https://doi.org/40.1016j.crhy.2010.04.002 }\end{array}$ \\
\hline Issue Date & 2010-08 \\
\hline Doc URL & http://hdl.handle.net/2115/47504 \\
\hline Type & article (author version) \\
\hline File Information & CRP11-7-8_421-432.pdf \\
\hline
\end{tabular}

Instructions for use 


\section{Emission of Terahertz Radiation from Two-Dimensional Electron Systems in Semiconductor Nano-Heterostructures}

Taiichi Otsuji ${ }^{\mathrm{a} *}$, Hiromi Karasawa ${ }^{\mathrm{a}}$, Takayuki Watanabe ${ }^{\mathrm{a}}$, Tetsuya Suemitsu ${ }^{\mathrm{a}}$, Maki Suemitsu ${ }^{\mathrm{a}}$, Eiichi Sano ${ }^{\mathrm{b}}$, Wojciech Knap ${ }^{\mathrm{c}}$, and Victor Ryzhii ${ }^{\mathrm{d}}$

${ }^{a}$ Research Inst. of Electrical Communication, Tohoku University, Sendai, Japan

${ }^{\mathrm{b}}$ Research Center for Integrated Quantum Electronics, Hokkaido University, Sapporo, Japan

${ }^{c}$ University of Montpellier \& Centre National de la Recherche Scientifique, Montpellier, France

${ }^{\mathrm{d}}$ Computational Nano-Electronics Laboratory, University of Aizu, Aizu-Wakamatsu, Japan

* Corresponding author

Email: otsuji@riec.tohoku.ac.jp, karasawa@riec.tohoku.ac.jp,watanabe@ riec.tohoku.ac.jp, sue@ @riec.tohoku.ac.jp, suemitsu@ riec.tohoku.ac.jp, esano@ rciqe.hokudai.ac.jp, knap@univ-montp2.fr, v-ryzhii@u-aizu.ac.jp 


\begin{abstract}
This paper reviews recent advances in emission of terahertz radiation from two-dimensional (2D) electron systems in semiconductor nano-heterostructures. 2D plasmon resonance is first presented to demonstrate intense broadband terahertz emission from InGaP/InGaAs/GaAs material systems. The device structure is based on a high-electron mobility transistor and incorporates the author's original interdigitated dual-grating gates. Second topic focuses on graphene, a monolayer carbonatomic honeycomb lattice crystal, exhibiting unique carrier transport and optical properties owing to massless and gapless energy spectrum. Coherent stimulated terahertz emission from femtosecond infrared-laser pumped epitaxial graphene is experimentally observed, reflecting the occurrence of negative dynamic conductivity and population inversion.
\end{abstract}

Keywords: Terahertz emission; Two-dimensional electrons; plasmons; graphene; heterostructures 


\section{INTRODUCTION}

In the research of modern terahertz (THz) electronics, development of compact, tunable and coherent sources operating at $\mathrm{THz}$ frequencies is one of the hottest issues [1]. Two-dimensional (2D) plasmons in semiconductor nano-hetero-structures like electron channels in high-electron mobility transistors (HEMT's) have attracted much attention due to their nature of promoting emission of electromagnetic radiation in the $\mathrm{THz}$ range. Therefore different devices/structures of micron and submicron sizes supporting low-dimensional plasmons were intensively studied as possible candidates for solid-state far-infrared (FIR)/THz sources [1-16]. Mechanisms of plasma wave excitation/emission can be divided (by convention) into two types: (i) incoherent and (ii) coherent type. The first is related to thermal excitation of broadband nonresonant plasmons by hot electrons [2-7]. The second is related to the plasma wave instability mechanisms like Dyakonov-Shur Doppler-shift model $[8,16]$ and/or Ryzhii-Satou-Shur transit-time model [17, 18], where coherent plasmons can be excited either by hot electrons or by optical phonon emission under near ballistic electron motion [19].

On the other hand, graphene, a monolayer of carbon atoms in a honeycomb lattice crystal, has attracted considerable attention due to its unique carrier transport and optical properties, including massless and gapless energy spectra [20-22]. The gapless and linear energy spectra of electrons and holes lead to nontrivial features such as negative dynamic conductivity. Ryzhii et al. analytically revealed that optically pumped graphene can exhibit negative-dynamic conductivity in the $\mathrm{THz}$ spectral range $[23,24]$ which may lead to the development of a new type of $\mathrm{THz}$ laser $[25,26]$.

To realize such graphene-based devices, understanding the non-equilibrium carrier relaxation/recombination dynamics is critical. Ultrafast scattering of photoexcited carriers by optical phonons has been theoretically predicted [27-29]. Recently, time-resolved measurements of fast non-equilibrium carrier relaxation dynamics have been carried out for multilayers and 
monolayers of graphene that were epitaxially grown on $\mathrm{SiC}$ [30-34] and exfoliated from highly oriented pyrolytic graphite (HOPG) $[35,36]$. For the electron-hole recombination, radiative recombination via direct-transition may take place due to the gapless symmetrical band structure. Photon emissions over a wide $\mathrm{THz}$ frequency range are expected if the pumping photon energy is suitably chosen and the pumping intensity is sufficiently high. This may lead to the abovementioned laser operation if the graphene is accommodated in a high quality factor cavity. This paper reviews recent advances in emission of terahertz radiation from $2 \mathrm{D}$ electron systems in IIIV HEMT's and graphene semiconductor nano-heterostructures.

\section{THz Emission from 2D Plasmon Resonance}

\subsection{Device Structure and Performance}

We are proposing our original 2D-plasmon-resonant micro-chip emitter as a new terahertz light source [37-42]. Figure 1 illustrates the cross section of the plasmon-resonant emitter [37]. The device structure is based on a HEMT and incorporates (i) interdigitated dual-grating gates (G1 and G2) that periodically localize the 2D plasmon in stripes on the order of $100 \mathrm{~nm}$ with a micron-to-submicron interval and (ii) a vertical cavity structure in between the top grating plane and a $\mathrm{THz}$ mirror at the backside. The structure (i) works as a $\mathrm{THz}$ antenna and (ii) works as an amplifier.

When the DC drain-to-source bias $V_{\mathrm{DS}}$ is applied, 2D electrons are accelerated to produce a constant drain-to-source current $I_{\mathrm{DS}}$. Due to such a distributed plasmonic cavity systems in periodic 2D electron-density modulation, the DC current flow may excite the plasma waves in each plasmonic cavity. As shown in Fig. 2, asymmetric cavity boundaries make plasma-wave reflections as well as fractions in the density and the drift velocity of electrons, which may cause the current-driven plasmon instability $[8,12,17,18]$ leading to excitation of coherent resonant plasmons. Thermally excited hot electrons also may excite incoherent plasmons $[4,7,40]$. The 
grating gates act also as $\mathrm{THz}$ antenna that converts non-radiative longitudinal plasmon modes to radiative transverse electromagnetic modes $[2,3,6,12,37]$.

Once the $\mathrm{THz}$ electromagnetic waves are produced from the seed of plasma waves, downward-propagating electromagnetic waves are reflected at the mirror back to the plasmon region so that the reflected waves can directly excite the plasmon again according to the Drude optical conductivity [37]. When the plasmon resonant frequency satisfies the standing-wave condition of the vertical cavity, the $\mathrm{THz}$ electromagnetic radiation will reinforce the plasmon resonance in a recursive manner. Therefore, the vertical cavity may work as an amplifier of $\mathrm{THz}$ radiation if the gain exceeds the cavity loss. The quality factor of the vertical cavity is relatively low, but as simulated in [37], it can serve as a broadband response amplifier.

The experimentally investigated device was fabricated with $\mathrm{InGaP} / \mathrm{InGaAs} / \mathrm{GaAs}$ material systems in a double-deck HEMT with semiconducting 2D electron gas (2DEG) grating gates [41, 42]. The schematic device cross section and its SEM image are shown in Fig. 3. The 2D plasmon layer was formed with a lower-deck quantum well at the heterointerface between a 15-nm thick InGaAs channel layer and a 60-nm thick, InGaP carrier-supplying layer. The upper-deck InGaAs channel, was serving as the dual grating-gate electrodes. It was periodically etched to form the uncapped region where the 2DEG concentration becomes lower than the capped region modulating the carrier density in this way without any external gate bias. For the source/drain ohmic contacts, AuGe/Ni was lifted off and annealed after the upper-deck HEMT was selectively etched. The intrinsic device area has geometry of $30 \mu \mathrm{m} \times 75 \mu \mathrm{m}$, where the grating pattern is replicated on the upper-deck HEMT layer. The grating consisted of 150-nm lines and 1850-nm lines aligned alternately with a spacing of $100 \mathrm{~nm}$. The number of fingers was 37 (38) for the 1500-nm (1850-nm) grating. The substrate thickness was $260 \mu \mathrm{m}$, corresponding to the fundamental vertical cavity resonance of $80 \mathrm{GHz}$ and the odd harmonics with a 160-GHz spacing. 
The field emission property of the fabricated chip was characterized by using a Fouriertransformed far-infrared spectrometer (FTIR) [42]. The microchip emitter die was mounted on a quartz substrate. The contact pads on the die were electrically interconnected to the biasing metal lines patterned on the substrate via gold bonding wires. The substrate was attached into a dedicated sample holder. Then the sample holder was installed into the FTIR source chamber. The electromagnetic radiation from the device was introduced to the Martin-Pupplett interferometer room and led to a liquid-He cooled $\mathrm{Si}$ bolometer. The responsivity and noiseequivalent power (NEP) of the detector were $2.84 \times 10^{5} \mathrm{~V} / \mathrm{W}^{-1}$ and $1.16 \times 10^{-13} \mathrm{~W} / \mathrm{Hz}^{-1 / 2}$, respectively. The experimental procedure was as follows; first the background radiation under turning-off the device was measured, then the radiation with device turned on was measured. The spectrum of the first-step measurement contained the $300 \mathrm{~K}$ blackbody radiation modified by the spectral functions of all the elements inside the chamber. Thus, in order to extract the contents of the real emission by the active operation of the device, the spectrum of the second-step measurement was normalized to the first-step one.

As shown in Fig. 4(a), the device was emitting 0.5-to-6.5 THz radiation at $300 \mathrm{~K}$ under appropriate bias conditions. The maximum emission power at $V_{\mathrm{DS}}=12.0 \mathrm{~V}$ was estimated to be of the order of $1 \mu \mathrm{W}$ at $300 \mathrm{~K}$. Taking into account the monitored power consumption of the order of $100 \mathrm{~mW}$, the energy conversion efficiency (from $\mathrm{DC}$ to $\mathrm{THz}$ ) was estimated to be of the order of $10^{-5}$. Such a broadband emission with threshold/super-linear dependence on the drain bias is analytically interpreted as the excitation of multimode plasmons: thermal excitation of incoherent plasmons $[2,43]$ as well as coherent plasmons related to instability-driven and/or transit-timedriven self-excitation $[8,44]$. We attribute the fine spectral structure to longitudinal-mode-like vibrations due to substrate-induced Fabry-Perot modes. The substrate thickness of $260 \mu \mathrm{m}$ gives the longitudinal modes (odd harmonics) in a $160-\mathrm{GHz}$ distance, which perfectly coincides with 
the observed periodicity of the fine spectral modes. Since the plasmonic cavity must have high emissivity of its main role, the reflection coefficient of the plasmonic plane is relatively low. This results in an insufficient quality factor of the vertical cavity, leading to such a broadband emission. Coherent monochromatic emission can be realized by engineering/accommodating high-Q cavity and/or by injection locking of the resonant frequency that can be produced for example by the photomixing of two infrared/visible lasers [45].

The emission spectral profile of the fabricated device was compared to that of a standard water-cooled high-pressure mercury lamp used as a standard THz light source in FTIR systems. Fig 4(a) plots the typical results [42]. It is clearly seen that the main lobe of the emission spectra of the fabricated device stays around 1 to $6 \mathrm{THz}$. The emission spectrum of the mercury lamp traces the typical black-body radiation curve. Its emission weakens substantially at lower $\mathrm{THz}$ region.

Emission power intensity of the mercury lamp in the $\mathrm{THz}$ region of our interest is about 30 times higher than that for a single-chip plasmon emitter at the sacrifice of huge power consumption (three orders of magnitude higher than that for a single-chip HEMT emitter). It is possible for the plasmon emitters to easily boost the emission power by orders of magnitude with reasonable power consumption by implementing in an arrayed structure. Preliminary trial of multi-chip operation was carried out [42]. The wire-bonded two independent emitters fabricated on a single wafer were electrically biased at $V \mathrm{ds}=9.0 \mathrm{~V}$ to operate simultaneously. Figure 4(b) demonstrates that the dual-chip operations can almost double the emission intensity. It is also worth to note that the plasmon emitter device may operate without any water cooling facility. From the above investigation, the plasmon-resonant emitter has a competitive potentiality to replace the standard $\mathrm{Hg}$ lamp sources in the $\mathrm{THz}$ spectroscopic measurement.

\subsection{Application to $\mathrm{THz}$ Spectroscopy}


The fabricated sample was introduced to a FTIR system as a 'microchip' THz source and used in a few standard spectroscopy tests. Water-vapor absorption spectrum was successfully observed at 300K, and compared to the standard data provided by NASA (see Fig. 5) [42]. Also the transmission spectra of two different types of sugar groups were measured: honey and maple syrup, both of which contain their finger prints in the $\mathrm{THz}$ region. The measured spectra were compared with the results of the data base of RIKEN (see Fig. 6). The results exhibit fairly identical spectral features confirming potential of the HEMTs plasma sources [42].

The results using a standard high-pressure mercury lamp as the light source are also plotted in Fig. 6. Due to its intense emission (by one order of magnitude higher than that for the single microchip emitter), excellent $\mathrm{S} / \mathrm{N}$ is obtained. Preliminary trial of dual chip operation of the microchip emitter successfully doubles the output intensity over the emission band [42]. Thus, we predict that an arrayed integration of the emitter can improve the emission intensity by orders of magnitude. To the best of the authors' knowledge, this is the first-time demonstration of $\mathrm{THz}$ sensing/spectroscopic applications utilizing room-temperature operating $\mathrm{THz}$ semiconductor microscopic light emitters. Future improvements of the emission power may enable this device to be a candidate as a new $\mathrm{THz}$ light source competing with standard black body radiation based ones.

\section{Stimulated THz Emission from Optically-Pumped Graphene}

\subsection{Carrier Dynamics of Optically Pumped Graphene}

When graphene is pumped with photon energy $\hbar \Omega$, electrons and holes are photogenerated via interband transitions. Figure 7 presents the carrier relaxation/recombination processes and the non-equilibrium energy distributions of photoelectrons/photoholes at specific times from $\sim 10 \mathrm{fs}$ to few picoseconds after the pumping event [23]. Usually photoexcited carriers are first cooled and thermalized mainly by intraband relaxation processes on femtosecond to subpicosecond time 
scales, and then by interband recombination processes. A recent study by Breusing et al. [36] precisely revealed ultrafast carrier dynamics for exfoliated graphene and graphite with a time resolution as low as $10 \mathrm{fs}$. It has been shown that the intraband carrier equilibration in optically excited graphene (with pumping photon energy $\hbar \Omega$ ) first establishes separate quasi-equilibrium distributions of electrons and holes at around the level $\varepsilon_{f} \pm \hbar \Omega / 2\left(\varepsilon_{f}\right.$ : Fermi energy) within 2030 fs after excitation (see Fig. 7(b)), followed by cooling of these electrons and holes by emission of a cascade $\left(N\right.$ times) of optical phonons $\left(\hbar \omega_{0}\right)$ within $200 \mathrm{fs}$ time scale. After this process these electrons and holes occupy the states $\varepsilon_{f} \pm \varepsilon_{N} \approx \varepsilon_{f} \pm \hbar\left(\Omega / 2-N \omega_{0}\right), \varepsilon_{N}<\hbar \omega_{0}$ (see Fig. 7(c)). Then, thermalization can occur via electron-hole recombination as well as intraband Fermization due to carrier-carrier (cc) scattering and carrier-phonon (cp) scattering (as shown with energy $\hbar \omega_{q}$ in Fig. 7(a)). This happens on a picosecond time scale (see Fig. 7(d)), because the interband cc scattering and cp scattering are slowed by the density of states effects and Pauli blocking. As a consequence, photoelectrons/holes recombine to radiate $\mathrm{THz}$ photons with the energy $\hbar \omega \approx 2 \varepsilon_{N}$ as shown in Fig. 7(a) [23].

Let us consider the $\mathrm{THz}$ optical conductivity due to such a relaxation/recombination processes responsible for the carriers staying at $\varepsilon_{f} \pm \hbar \omega / 2$. The electron and hole distribution functions at the Dirac point are $f_{e}(0)=f_{h}(0)=1 / 2$. This implies that at even weak photoexcitation, the values of the distribution functions at low energies $\varepsilon$ can be $f_{e}(\varepsilon)=f_{h}(\varepsilon)>1 / 2$, that corresponds to the population inversion [23]. Such a population inversion might lead to the interband transitions related negative ac conductivity at $\mathrm{THz}$ frequencies. However, the intraband processes determined by the Drude conductivity provide the positive contribution to the ac conductivity. One might expect that under sufficiently strong optical excitation resulting in photogeneration of electron-hole pairs, total ac conductivity becomes "negative". 
The real part of the net $a c$ conductivity $\operatorname{Re} \sigma_{\omega}$ is proportional to the absorption of photons with frequency $\omega$ and comprises the contributions of both interband and intraband transitions [23],

$$
\operatorname{Re} \sigma_{\omega}=\operatorname{Re} \sigma_{\omega}^{\text {inter }}+\operatorname{Re} \sigma_{\omega}^{\text {intra }} .
$$

Let us assume a relatively weak optical excitation: $\varepsilon_{F}<k_{B} T$, where $\varepsilon_{F}$ the non-equilibrium quasi-Fermi energy and $k_{B} T$ the thermal energy. In this case, for the $\mathrm{THz}$ frequencies $\omega<2 k_{B} T / \hbar, \operatorname{Re} \sigma_{\omega}^{\text {inter }}$ can be presented as

$$
\operatorname{Re} \sigma_{\omega}^{\text {inter }}=\frac{e^{2}}{4 \hbar}\left[1-f_{e}\left(\frac{\hbar \omega}{2}\right)-f_{h}\left(\frac{\hbar \omega}{2}\right)\right] \approx \frac{e^{2}}{8 \hbar}\left(\frac{\hbar \omega}{2 k_{B} T}-\eta_{F}\right),
$$

where $e$ the elementary charge, $\eta_{F} \equiv \varepsilon_{F} / k_{B} T$ the normalized quasi-Fermi energy [23]. On the other hand, Re $\sigma_{\omega}^{\text {intra }}$ can be presented as

$$
\operatorname{Re} \sigma_{\omega}^{i n t r a} \approx \frac{\left(\ln 2+\eta_{F} / 2\right) e^{2}}{\pi \hbar} \frac{T \tau}{\hbar\left(1+\omega^{2} \tau^{2}\right)},
$$

where $\tau$ the momentum relaxation time of electrons/holes [23]. Since $\eta_{F}$ corresponds to the ratio of the excess electron/hole concentration $\delta n$ (equivalent to photoelectron/photohole concentration) to the thermally equilibrium electron/hole concentration $n_{0}, \eta_{F}$ is given by

$$
\eta_{F}=\frac{\delta n}{n_{0}}=\frac{\tau_{R} \alpha_{\Omega} I_{\Omega}}{n_{0}} \approx \frac{12 e^{2}}{\hbar c}\left(\frac{\hbar v_{F}}{k_{B} T}\right)^{2} \frac{\tau_{R} I_{\Omega}}{\hbar \Omega},
$$

where $\tau_{R}$ electron/hole recombination time, $\alpha_{\Omega}$ the interband absorption coefficient, $I_{\Omega}$ the pumping intensity, $c$ the speed of light, $v_{F}$ the Fermi velocity [23].

As a consequence, $\operatorname{Re} \sigma_{\omega}$ becomes 


$$
\begin{aligned}
\operatorname{Re} \sigma_{\omega} & \approx \frac{e^{2}}{8 \hbar}\left[\frac{\hbar \omega}{2 k_{B} T}+\frac{8 \ln 2}{\pi} \frac{k_{B} T \tau}{\hbar\left(1+\omega^{2} \tau^{2}\right)}-\frac{12 e^{2}}{\hbar c}\left(\frac{\hbar v_{F}}{k_{B} T}\right)^{2} \frac{\tau_{R} I_{\Omega}}{\hbar \Omega}\right] \\
& =\frac{e^{2} \bar{g}}{8 \hbar}\left[1+\frac{3}{2}\left(\frac{\omega-\bar{\omega}}{\bar{\omega}}\right)^{2}-\frac{I_{\Omega}}{\overline{I_{\Omega}}}\right]
\end{aligned}
$$

where

$$
\bar{\omega} \approx\left(\frac{k_{B} T \tau}{\hbar}\right)^{2 / 3} \frac{1.92}{\tau}, \overline{I_{\Omega}} \approx 11\left(\frac{\hbar}{k_{B} T \tau}\right)^{1 / 3}\left(\frac{k_{B} T}{\hbar v_{F}}\right)^{2} \frac{\hbar \Omega}{\tau_{R}}
$$

When the pumping intensity exceeds the threshold: $I_{\Omega}>\overline{I_{\Omega}}, \operatorname{Re} \sigma_{\omega}$ becomes negative in a certain range around $\bar{\omega}$. When $T=300 \mathrm{~K}, \tau=10^{-12} \mathrm{~s}, \quad \tau_{R}=10^{-9} \square 10^{-11} \mathrm{~s}, \bar{I}_{\Omega} \approx 60 \sim 6000$ $\mathrm{W} / \mathrm{cm}^{2}$. Assuming the device size of $100 \mu \mathrm{m} \times 100 \mu \mathrm{m}$, we find that the required pumping intensity, which provides the negative dynamic conductivity, $\bar{I}_{\Omega} \approx 6 \sim 600 \mathrm{~mW}$.

Fig. 8 plots the calculated $\operatorname{Re} \sigma_{\omega}$ for various pumping intensities when $\mathrm{T}=300 \mathrm{~K}$, $\tau=10^{-12} \mathrm{~s}$, and $\tau_{R}=10^{-9} \mathrm{~s}$. The vertical scale is normalized to the characteristic conductivity $e^{2} / 2 \hbar$. It is clearly seen that the frequency range where the conductivity becomes negative widens with pumping intensity.

\subsection{Observation of Stimulated $\mathrm{THz}$ Emission}

In order to verify the proposed concept, we conduct an experimental study on the electromagnetic radiation emitted from an optically pumped graphene structure using $\mathrm{THz}$ emission spectroscopy [46]. The sample used in this experiment is heteroepitaxial graphene film grown on a $\mathrm{SiC}(110)$ thin film heteroepitaxially grown on a $\mathrm{Si}(110)$ substrate via thermal graphitization of the SiC surface [47-49]. In the Raman spectrum of the graphene film, the principal bands of graphene, namely, the $G\left(1595 \mathrm{~cm}^{-1}\right)$ and $\mathrm{G}^{\prime}\left(2730 \mathrm{~cm}^{-1}\right)$ bands, are observed 
as shown in Fig. 9. Furthermore, as is shown in Fig. 9, transmission electron microscopy images indicate that the film is stratified. It is thus concluded that epitaxial graphene with a planar structure can be produced by this fabrication method. Furthermore, the epitaxial graphene layer is inferred to have a non-Bernal stacking arrangement because the G' band in the Raman spectrum can be expressed as a single component related to the two-dimensionality of the graphene film $[50,51]$. The non-Bernal stacked epitaxial graphene layers grown by our method can be treated as a set of isolated single graphene layers, as in the case of an epitaxial graphene layer on a Cterminated SiC bulk crystal [52]. The G-band peak at $1595 \mathrm{~cm}^{-1}$ corresponds to an optical phonon energy at the zone center of $197.8 \mathrm{meV}$.

We measure the carrier relaxation and recombination dynamics in optically pumped epitaxial graphene-on-silicon (GOS) heterostructures using THz time-domain spectroscopy based on an optical pump/THz-and-optical-probe technique. The time-resolved field emission properties are measured by an electro-optic sampling method in total-reflection geometry [53]. To obtain the $\mathrm{THz}$ photon emissions from the above-mentioned carrier relaxation/recombination dynamics, the pumping photon energy (wavelength) is carefully selected to be around $800 \mathrm{meV}(1550 \mathrm{~nm})$. To perform intense pumping beyond the threshold, a femtosecond pulsed fiber laser with full width at half-maximum (FWHM) of $80 \mathrm{fs}$, pulse energy of $50 \mathrm{pJ} /$ pulse, and frequency of $20 \mathrm{MHz}$ was used as the pumping source. The setup is shown in Fig. 10. The graphene sample is placed on the stage and a 100- $\mu$ m-thick (101)-oriented CdTe crystal is placed onto the sample; the CdTe crystal acts as a THz probe pulse emitter as well as an electro-optic sensor. The single femtosecond fiber laser beam is split into two beams: one for optical pumping and generating the THz probe beam, and one for optical probing. The pumping laser, which is linearly polarized and mechanically chopped at $\sim 1.2 \mathrm{KHz}$, is simultaneously focused at normal incidence onto the sample and the CdTe from below, while the probing laser, which is cross-polarized to the pumping beam, is focused from above. The incident pumping beam is defocused on the sample to satisfy the above- 
mentioned pumping power requirements. The resulting photoexcited carrier density is $\sim 8 \times 10^{10} \mathrm{~cm}^{-2}$, which is comparable to the background carrier density. Owing to second-order nonlinear optical effects, the CdTe crystal can rectify the pumping laser pulse to emit $\mathrm{THz}$ envelope radiation. Such THz pulses irradiate the graphene sample, acting as $\mathrm{THz}$ probe signals to stimulate $\mathrm{THz}$ photon emission via electron-hole recombination in the GOS. Due to the geometrical situation of the experimental setup as shown in Fig. 10, the time delay of the $\mathrm{THz}$ probe with respect to optical pumping is fixed at around 200-300 fs so that the photoelectrons/holes are stimulated immediately after losing their energy via the cascade of optical phonon emissions, when they still have a distribution of $\varepsilon_{f} \pm \varepsilon_{N}$ (as is shown in Fig. 7(c)).

On the other hand, through the Si prism attached to the CdTe crystal, the optical probing beam is totally reflected back to the lock-in detection block, and its phase information reflecting the electric field intensity is lock-in amplified. By sweeping the timing of the optical probe using an optical delay line, the whole temporal profile of the field emission properties can be obtained. The system bandwidth is estimated to be around $6 \mathrm{THz}$, which is limited mainly by the Reststrahlen band of the CdTe sensor crystal.

Figure 11 shows the autocorrelations and spectral profiles of the pumping laser beams under two different pulse compression conditions. The horizontal axis indicates the wavelength and frequency together with the estimated $\mathrm{THz}$ photon frequency to be emitted from the sample. The dotted line plots the dynamic conductivity at a pumping intensity twice as high as the threshold intensity calculated for $300 \mathrm{~K}$ using Eqs. (1)-(3) with a $\tau$ value of $1 \times 10^{-12} \mathrm{ps}$. The shaded area shows the negative dynamic conductivity.

First, the experiment was conducted with the pumping pulse shown in Fig. 11 as red lines. Figure 12(a) shows the measured results. The emission from the CdTe without graphene exhibits a temporal response similar to optical rectification with a single peak at around $1 \mathrm{THz}$ and an 
upper weak side lobe extending to around $7 \mathrm{THz}$ (blue lines in Fig. 12). On the other hand, the results with graphene agree well with the pumping photon spectrum and include an additional peak around $1 \mathrm{THz}$ from the original CdTe spectrum (red lines in Fig. 12). It is thought that the $\mathrm{THz}$ emissions from graphene are stimulated by the coherent $\mathrm{THz}$ probe radiation that originates from the CdTe excited by the pump laser beam. The $\mathrm{THz}$ emissions are amplified by photoelectron/hole recombination in the range of the negative dynamic conductivity.

In order to confirm with certainty that the emissions from graphene reflect the pumping photon spectrum, the pulse compression of the pumping laser pulse was altered to broaden its spectrum as shown by the black lines in Fig. 11. Due to the pulse compression condition, the pulse energy increases $\sim 100 \mathrm{pJ} /$ pulse. As can be seen in Fig. 12(b), the emission spectrum of the graphene was broadened, corresponding to the pumping photon energy.

Furthermore, to confirm the effects of the $\mathrm{THz}$ probe, we replace the first CdTe crystal with another CdTe crystal having a high-reflectivity coating for IR on its bottom surface, in order to eliminate generation of the $\mathrm{THz}$ probe signal. In this case, no distinctive response is observed with or without graphene. Since the measurements are taken as an average, the observed response is undoubtedly a coherent process that cannot be obtained via spontaneous emission processes, providing clear evidence of stimulated emission. For all three cases of emissions from graphene in Figs. 12(a) and (b), the lower cutoff around $2 \mathrm{THz}(8.3 \mathrm{meV})$ is slightly higher than the theoretical estimation $(\sim 5 \mathrm{meV})$ [23]. This may be due to a small bandgap forming due to the interlayer coupling of the existing multilayer graphene or substrate-induced asymmetric potential deformation [54].

From the above results, it is inferred that $\mathrm{THz}$ emissions from graphene are stimulated by the coherent $\mathrm{THz}$ probe radiation. Furthermore, the $\mathrm{THz}$ emissions are amplified via photoelectron/hole recombination in the range of the negative dynamic conductivity. In conclusion, we have successfully observed coherent amplified stimulated $\mathrm{THz}$ emissions arising 
from the fast relaxation and relatively slow recombination dynamics of photogenerated electrons/holes in epitaxial graphene heterostructures. The results provide evidence of the occurrence of negative dynamic conductivity, which can potentially be applied to a new type of $\mathrm{THz}$ laser.

\section{Conclusion}

Recent advances in emission of terahertz radiation from $2 \mathrm{D}$ electron systems in semiconductor nano-heterostructures were reviewed. 2D plasmon resonance in HEMT structures as well as ultrafast non-equilibrium dynamics of massless electrons/ holes in graphene are promising mechanisms for making new types of practical THz solid-state light sources.

\section{Acknowledgements}

The author would like to thank Tsuneyoshi Komori, Yuki Tsuda, Hiroyuki Handa, Yu Miyamoto, and Hirokazu Fukidome at RIEC, Tohoku University, Japan, Yahya M. Meziani at Salamanca University, Spain, Viacheslav Popov at KIREE, Russian Academy of Science, Saratov, Russia, Dominique Coquillat, Abdelouahad El Fatimy, and Frederic Teppe at CNRSUniversity of Montpellier, France, and Maxim Ryzhii and Akira Satou at University of Aizu, Japan, for their extensive contributions throughout this work. This work is financially supported in part by the JST-CREST, Japan, and the JSPS-BR(S), Japan.

\section{References}

[1] M. Tonouchi, Cutting-edge terahertz technology, Nature Photon. 1 (2007) 97-105.

[2] R. A. Hopfel, E. Vass, and E. Gornik, Thermal Excitation of Two-Dimensional Plasma Oscillations, Phys. Rev. Lett. 49 (1982) 1667-1671. 
[3] D. C. Tsui, E. Gornik and R. A. Logan, Far infrared emission from plasma oscillations of Si inversion layers, Solid State Communications. 35 (1980) 875-877.

[4] N. Okisu, Y. Sambe, and T. Kobayashi, Far-infrared emission from two-dimensional plasmons in AlGaAs/GaAs heterointerfaces, Appl. Phys. Lett. 48 (1986) 776-778.

[5] R. Hopfel, G. Lindemann, E. Gornik, G. Stangl, A. C. Gossard and W. Wiegmann, Cyclotron and plasmon emission from two-dimensional electrons in GaAs, Surf. Sci. 113 (1982) 118-123.

[6] R.J. Wilkinson, C.D. Ager, T. Duffield, H.P. Hughes, D.G. Hasko, H. Armed, J.E.F. Frost, D.C. Peacock, D.A. Ritchie, A.C. Jones, C.R. Whitehouse, and N. Apsley, Plasmon excitation and self-coupling in a bi-periodically modulated two-dimensional electron gas, J. Appl. Phys. 71 (1992) 6049-6061.

[7] K. Hirakawa, K. Yamanaka, M. Grayson and D. C. Tsui, Far-infrared emission spectroscopy of hot two-dimensional plasmons in $\mathrm{Al}_{0.3} \mathrm{Ga}_{0.7} \mathrm{As} / \mathrm{GaAs}$ heterojunctions, Appl. Phys. Lett. 67 (1995) 2326-2328.

[8] M. Dyakonov and M. Shur, Shallow water analogy for a ballistic field effect transistor: New mechanism of plasma wave generation by dc current, Phys. Rev. Lett. 71 (1993) 2465-2468.

[9] W. Knap, J. Lusakowski, T. Parenty, S. Bollaert, A. Cappy, V. V. Popov, and M. S. Shur, Terahertz emission by plasma waves in $60 \mathrm{~nm}$ gate high electron mobility transistors, Appl. Phys. Lett. 84 (2004) 2331-2333.

[10]J. Lusakowski, W. Knap, N. Dyakonova, and L. Varani, Voltage tunable terahertz emission from a ballistic nanometer InGaAs/InAlAs transistor, J. Appl. Phys. 97 (2005) 064307.

[11]N. Dyakonova, F. Teppe, J. Lusakowski, W. Knap, M. Levinshtein, A. P. Dmitriev, M. S. Shur, S. Bollaert, and A. Cappy, Magnetic field effect on the terahertz emission from nanometer InGaAs/AlInAs high electron mobility transistors, J. Appl. Phys. 97 (2005) 114313.

[12]S.A. Mikhailov, Plasma instability and amplification of electromagnetic waves in lowdimensional electron systems, Phys. Rev. B. 58 (1998) 1517-1532. 
[13]P. Bakshi, K. Kempa, A. Scorupsky, C. G. Du, G. Feng, R. Zobl, G. Strasser, C. Rauch, Ch. Pacher, K. Unterrainer, and E. Gornik, Plasmon-based terahertz emission from quantum well structures, Appl. Phys. Lett. 75 (1999) 1685-1687.

[14]R. Colombelli, F. Capasso, C. Gmachl, A. L. Hutchinson, D. L. Sivco, A. Tredicucci, M. C. Wanke, A. M. Sergent, and A. Y. Cho, Far-infrared surface-plasmon quantum-cascade lasers at $21.5 \mu \mathrm{m}$ and $24 \mu \mathrm{m}$ wavelengths, Appl. Phys. Lett. 78 (2001) 2620-26202.

[15]A. Tredicucci, R. Kohler, L. Mahler, H. E. Beere, E. H. Linfield, and D. A. Ritchie, Terahertz quantum cascade lasers - first demonstration and novel concepts, Semicond. Sci. Technol. 20 (2005) S222-S227.

[16]A. El Fatimy, N. Dyakonova, Y. Meziani, T. Otsuji, W. Knap, S. Vandenbrouk, K. Madjour, D. Théron, C. Gaquiere, M. A. Poisson, S. Delage, P. Prystawko, and C. Skierbiszewski, $\mathrm{AlGaN} / \mathrm{GaN}$ high electron mobility transistors as a voltage-tunable room temperature terahertz sources, J. Appl. Phys. 107, pp. 024504, 2010. doi:10.1063/1.3291101

[17]V. Ryzhii, A. Satou, and M. Shur, Plasma Instability and Terahertz Generation in HEMTs Due to Electron Transit-Time Effect, IEICE Trans. Electron. E89-C (2006) 1012-1019.

[18]V. Ryzhii, A. Satou, M. Ryzhii, T. Otsuji, and M. S. Shur, Mechanism of self-excitation of terahertz plasma oscillations in periodically double-gated electron channels, J. Phys.: Condens. Matter 20 (2008) 384207.

[19]V. Ryzhij, N.A. Bannov, and V.A. Fedirko, "Ballistic and quasiballistic transport in semiconductor structures," Sov. Phys. Semicond.18 (1984) 481-491.

[20]A.K. Geim. K.S. Novoselov, The rise of graphene, Nat. Mat. 6 (2007) 183-191.

[21]K.S. Novoselov, A.K. Geim, S.V. Morozov, D. Jiang, M.I. Katsnelson, I.V. Grigorieva, S.V. Dubonos, A. Firsov, Two-dimensional gas of massless Dirac fermions in graphene, Nature 438 (2005) 197-200. 
[22]Y. Zhang, Y.-W. Tan, H. L. Stormer, P. Kim, Experimental observation of the quantum Hall effect and Berry's phase in graphene, Nature 438 (2005) 201-204.

[23]V. Ryzhii, M .Ryzhii, T. Otsuji, Negative dynamic conductivity of graphene with optical pumping, J. Appl. Phys. 101 (2007) 083114.

[24]V. Ryzhii, M .Ryzhii, T. Otsuji, Population inversion of photoexcited electrons and holes in graphene and its negative terahertz conductivity, Phys. Stat. Sol. (c) 5 (2008) 261-264.

[25]V. Ryzhii, M. Ryzhii, A. Satou, T. Otsuji, A. A. Dubinov and V. Y. Aleshkin, Feasibility of terahertz lasing in optically pumped epitaxial multiple graphene layer structures, J. Appl. Phys. 106 (2009) 084507.

[26]A. A. Dubinov, V. Y. Aleshkin, M. Ryzhii, T. Otsuji, and V. Ryzhii, Terahertz laser with optically pumped graphene layers and Fabri-Perot resonator, Appl. Phys. Express 2 (2009) 092301.

[27]T. Ando, Anomaly of optical phonon in monolayer graphene, J. Phys. Soc. Jpn. 75 (2006) 124701.

[28]H. Suzuura, T. Ando, Zone-boundary phonon in graphene and nanotube, J. Phys. Soc. Jpn. 77 (2008) 044703.

[29]F. Rana, P.A. George, J.H. Strait, J. Dawlaty, S. Shivaraman, M. Chandrashekhar, and M.G. Spencer, Carrier recombination and generation rates for intravalley and intervalley phonon scattering in graphene, Phys. Rev. B 79 (2009) 115447.

[30]J.M. Dawlaty, S. Shivaraman, M. Chandrashekhar, F. Rana, M.G. Spencer, Measurement of ultrafast carrier dynamics in epitaxial graphene, Appl. Phys. Lett. 92 (2008) 042116.

[31]D. Sun, Z.-K. Wu, C. Divin, X. Li, C. Berger, W.A. de Heer, P.N. First, T.B. Norris, Ultrafast Relaxation of Excited Dirac Fermions in Epitaxial Graphene Using Optical Differential Transmission Spectroscopy, Phys. Rev. Lett. 101 (2008) 157402. 
[32]P.A. George, J. Strait, J. Dawlaty, S. Shivaraman, M. Chandrashekhar, F. Rana, M.G. Spencer, Ultrafast Optical-Pump Terahertz-Probe Spectroscopy of the Carrier Relaxation and Recombination Dynamics in Epitaxial Graphene, Nano Lett. 8 (2008) 4248-4251.

[33]H. Choi, F. Borondics, D.A. Siegel, S.Y. Zhou, M.C. Martin, A. Lanzara, R.A. Kaindl, Broadband electromagnetic response and ultrafast dynamics of few-layer epitaxial graphene, Appl. Phys. Lett. 94 (2009) 172102.

[34]H. Wang, J.H. Strait, P.A. George, S. Shivaraman, V.B. Shields, M. Chandrashekhar, J. Hwang, F. Rana, M.G. Spencer, C.S. Ruiz-Vargas, and J. Park, Ultrafast relaxation dynamics of hot optical phonons in Graphene, Arxiv (2009) 0909.4912.

[35]T. Kampfrath, L. Perfetti, F. Schapper, C. Frischkorn, and M. Wolf, Strongly coupled optical phonons in the ultrafast dynamics of the electronic energy and current relaxation in graphite, Phys. Rev. Lett. 95 (2005) 187403.

[36]M. Breusing, C. Ropers, and T. Elsaesser, Ultrafast carrier dynamics in graphite, Phys. Rev. Lett. 102 (2009) 086809.

[37]T. Otsuji, M. Hanabe, T. Nishimura, E. Sano, A grating-bicoupled plasma-wave photomixer with resonant-cavity enhanced structure, Opt. Express 14 (2006) 4815-4825.

[38]T. Otsuji, Y.M. Meziani, M. Hanabe, T. Ishibashi, T. Uno, E. Sano, A grating-bicoupled plasmon-resonant terahertz emitter fabricated with GaAs-based heterostructure material systems, Appl. Phys. Lett. 89 (2006) 263502.

[39]T. Otsuji, Y .M. Meziani, M. Hanabe, T. Nishimura, E. Sano, Emission of terahertz radiation from InGaP/InGaAs/GaAs grating-bicoupled plasmon-resonant emitter, Solid State Electron. 51 (2007) 1319-1327.

[40]Y.M. Meziani, H. Handa, W. Knap. T. Otsuji, E. Sano, V.V. Popov, G.M. Tsymbalov, D. Coquillat, F. Teppe, Room temperature terahertz emission from grating coupled twodimensional plasmons, Appl. Phys. Lett. 92 (2008) 201108. 
[41]T. Otsuji, Y.M. Meziani, T. Nishimura, T. Suemitsu, W. Knap, E. Sano, T. Asano, V .V. Popov, Emission of terahertz radiation from dual-grating-gates plasmon-resonant emitters fabricated with InGaP/InGaAs/GaAs material systems, J. Phys.: Condens. Matter 20 (2008) 384206.

[42]Y. Tsuda, T. Komori, T. Watanabe, T. Suemitsu, T. Otsuji, Application of plasmonic microchip emitters to broadband terahertz spectroscopic measurement, J. Opt. Soc. Am. B 26 (2009) A52-A57.

[43]V.V. Popov, G.M. Tsymbalov, N.J.M. Horing, Anticrossing of plasmon resonances and giant enhancement of interlayer terahertz electric field in an asymmetric bilayer of two-dimensional electron strips, J. Appl. Phys. 99 (2006) 124303.

[44]V. Ryzhii, A. Satou, M .Ryzhii, T. Otsuji, M.S. Shur, Mechanism of self-excitation of terahertz plasma oscillations in periodically double-gated electron channels, J. Phys.: Condens. Matter 20 (2008) 384207.

[45]T. Otsuji, M. Hanabe and O. Ogawara, Terahertz plasma wave resonance of two-dimensional electrons in InGaP/InGaAs/GaAs high-electron mobility transistors, Appl. Phys. Lett. 85 (2004) 2119-2121.

[46]H. Karasawa, T. Komori, T. Watanabe, M. Suemitsu, V. Ryzhii, T. Otsuji, Observation of Carrier Relaxation and Recombination Dynamics in Optically Pumped Epitaxial Graphene Heterostructures Using Terahertz Emission Spectroscopy, Dig. CLEO-Europe, CF8.3, Munich, 2009.

[47]M .Suemitsu, Y. Miyamoto, H. Handa, A. Konno, Graphene formation on a 3C-SiC(111) thin film grown on Si(110) substrate, e-J. Surface Sci. Nanotech. 7 (2009) 311-313.

[48]Y. Miyamoto, H. Handa, E. Saito, A. Konno, Y. Narita, M. Suemitsu, H. Fukidome, T. Ito, K. Yasui, H. Nakazawa. T. Endoh, Raman-scattering spectroscopy of epitaxial graphene formed on SiC film on Si substrate, e-J. Surface Sci. Nanotech. 7 (2009) 107-109. 
[49]H. Fukidome, Y. Miyamoto, H. Handa, E. Saito, M. Suemitsu, Epitaxial Growth Processes of Graphene on Silicon Substrates, Jpn. J. Appl. Phys. in press.

[50]L.G. Cançadoa, K. Takaia, T. Enokia, M. Endob, Y.A. Kimb, H. Mizusakib, N.L. Spezialic, A. Jorioc and M.A. Pimentac, Measuring the degree of stacking order in graphite by Raman spectroscopy, Carbon 46 (2008) 272-275.

[51]C. Faugeras, A. Nerrière, M. Potemski, A. Mahmood, E. Dujardin, C. Berger, and W.A. de Heer, Few-layer graphene on $\mathrm{SiC}$, pyrolitic graphite, and graphene: A Raman scattering study, Appl. Phys. Lett. 92 (2008) 011914.

[52]J. Hass, F. Varchon, J.E. Millán-Otoya, M. Sprinkle, N. Sharma, W.A. de Heer, C. Berger, P.N. First, L. Magaud, and E.H. Conrad, Why multilayer graphene on 4H-SiC(0001) behaves like a single sheet of graphene, Phys. Rev. Lett. 100 (2008) 125504.

[53]L. Min, R.J.D. Miller, Sub-picosecond reflective electro-optic sampling of electronhole vertical transport in surface-space-charge field, Appl. Phys. Lett. 56 (1990) 524526.

[54]E. Sano, T. Otsuji, Theoretical evaluation of channel structure in graphene field-effect transistors , Jpn. J. Appl. Phys. 48 (2009) 041202. 


\section{Figure Captions}

Fig. 1. Device cross section for typical GaAs-based heterostructure material systems. Ex: the electric field (linear polarization), $k_{\mathrm{THz}}$ : the wave vector of electromagnetic radiation. (after Ref. 37.)

Fig. 2. Schematic band diagram and operation mechanism.

Fig. 3. Cross sectional and bird's view images of a fabricated plasmon-resonant emitter. (after Ref. 41.) The die mounted on a quartz substrate is also shown in comparison with a commercial high-pressure mercury lamp.

Fig. 4. FTIR measured emission spectra for a fabricated device in comparison with a highpressure mercury lamp. Double- and triple-chip operation almost doubles and triples the emission power, respectively. $\mathrm{V}_{\mathrm{DS}}=9.0 \mathrm{~V}$.

Fig. 5. Measured absorption spectrum of atmospheric water vapor in comparison with the data provided by NASA. (after Ref. 42.)

Fig. 6. Transmission spectra for sugar group samples. (a) Results for honey measured by using a single-chip plasmon-resonant emitter (PRE) and by using a high-pressure mercury lump, and their main ingredient(s) provided by RIKEN, (b) results for maple syrup measured by using sigleand triple-chip PRE(s), and by using a high-pressure mercury lump. 
Fig. 7. Schematic view of graphene band structure (a) and energy distributions of photogenerated electrons and holes (b)-(d). Arrows denote transitions corresponding to optical excitation by photons with energy $\hbar \Omega$, cascade emission of optical phonons with energy $\hbar \omega_{0}$, and radiative recombination with emission of photons with energy $\hbar \omega$. (b) after $\sim 20$ fs from optical pumping, (c) after 200 ps from optical pumping, (d) after 1 ps from optical pumping.

Fig. 8. Calculated $a c$ conductivity for various pumping intensities at $300 \mathrm{~K}$. The vertical scale is normalized to the characteristic conductivity $e^{2} / 2 \hbar$.

Fig. 9. Crystal properties of the fabricated heteroepitaxial graphene on silicon sample. Upper: photo and micro-zoom images, middle: TEM image, bottom: Raman spectra at D, G, and G' band.

Figure 10. Measurement setup for optical-pump/THz-and-optical-probe spectroscopy. A CdTe crystal on top of the graphene sample generates the $\mathrm{THz}$ probe pulse and electro-optically detects the THz electric field intensity.

Fig. 11. Temporal (autocorrelation) and spectral profiles of pumping laser beam for two pulse compression conditions used in this experiment. Dotted line denotes the dynamic conductivity normalized to the characteristic conductivity $e^{2} / 2 \hbar$ at a pumping intensity of twice the threshold intensity at $300 \mathrm{~K}$ calculated using Eqs. (1)-(3). Shaded area denotes negative dynamic conductivity.

Fig. 12. Measured field emission properties (inset: temporal responses; main plot: Fourier spectrum) when the $\mathrm{THz}$ prove beam is generated by optical rectification of pumping photons in CdTe. Dashed line is the photoemission spectrum predicted from the pumping laser spectrum. (a) 
Pumping with wider pulses (narrower spectrum) plotted with red curves in Fig. 11, and (b) pumping with shorter pulses (wider spectrum) plotted with black curves in Fig. 11. 
Fig. 1.

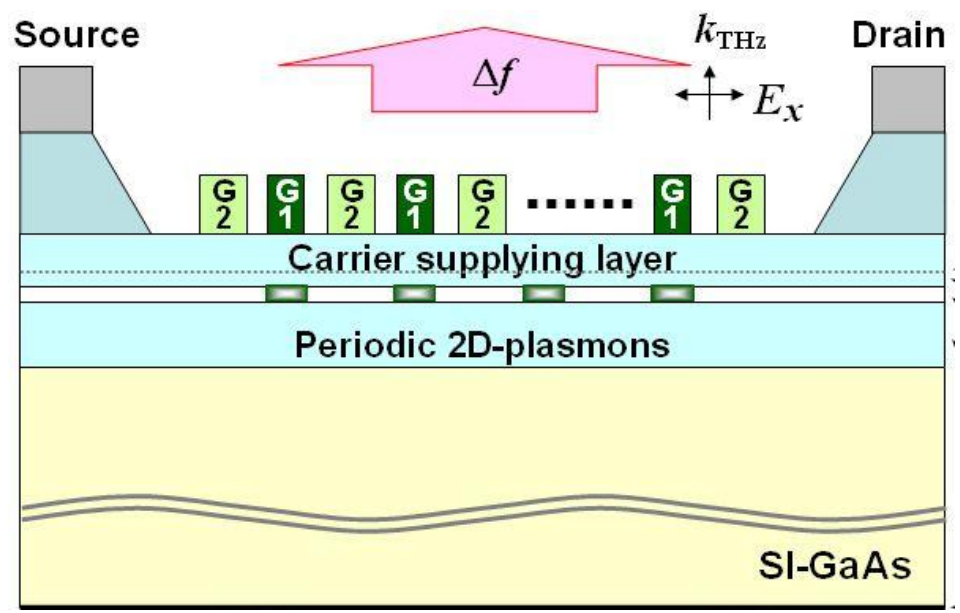


Fig. 2.

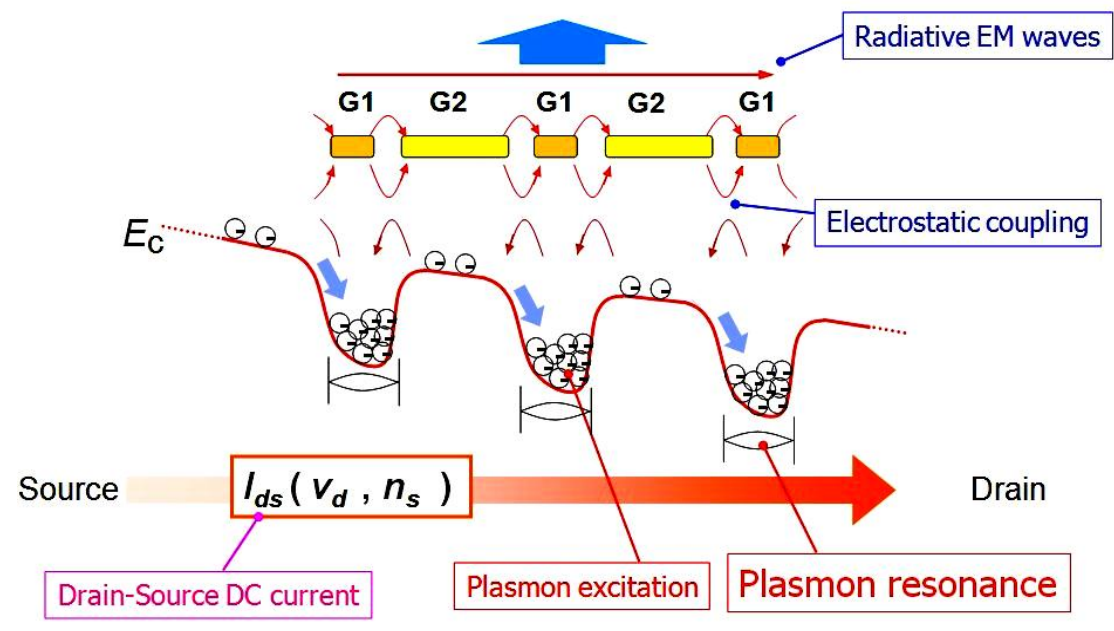


Fig. 3.
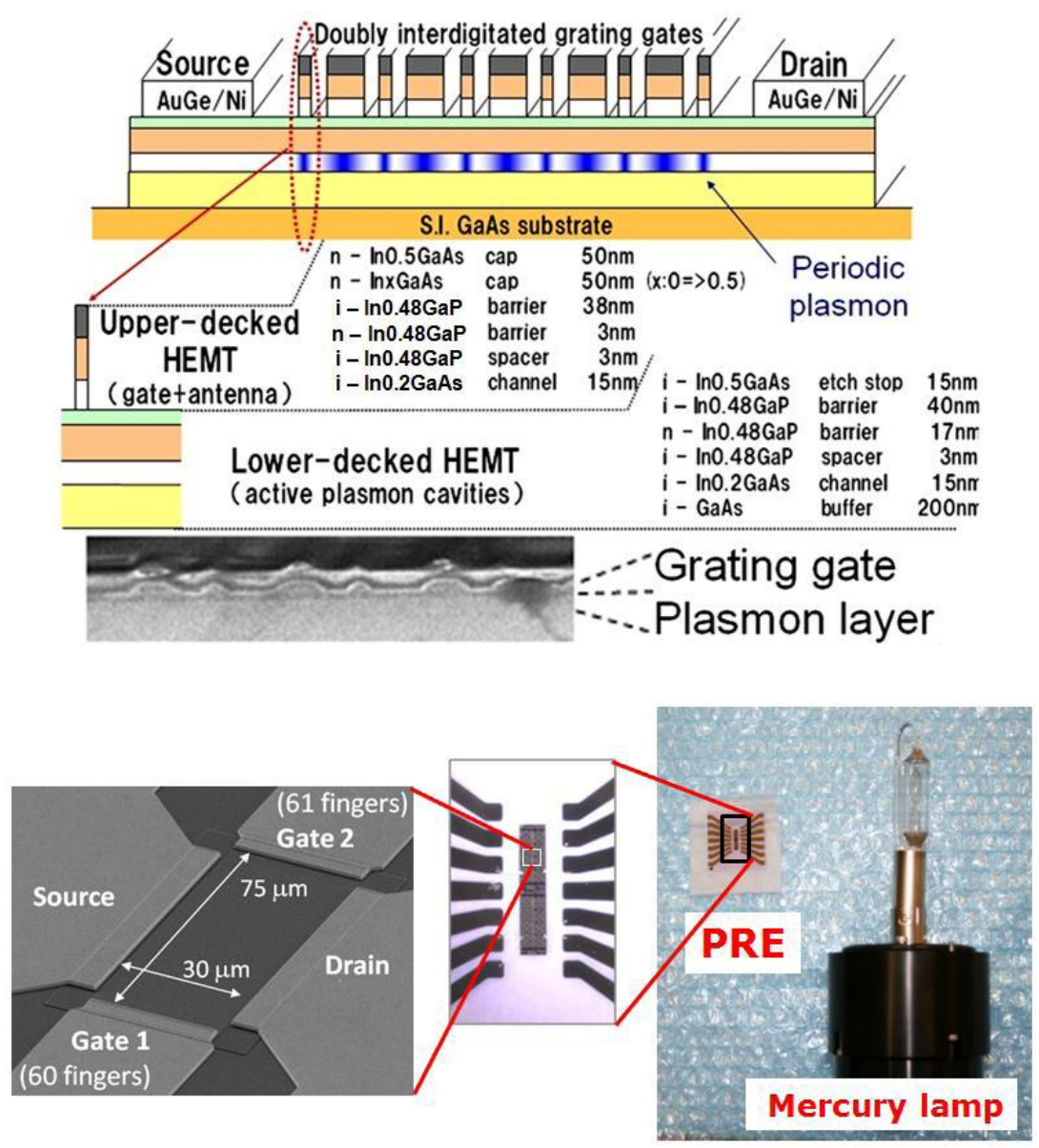
Fig. 4.

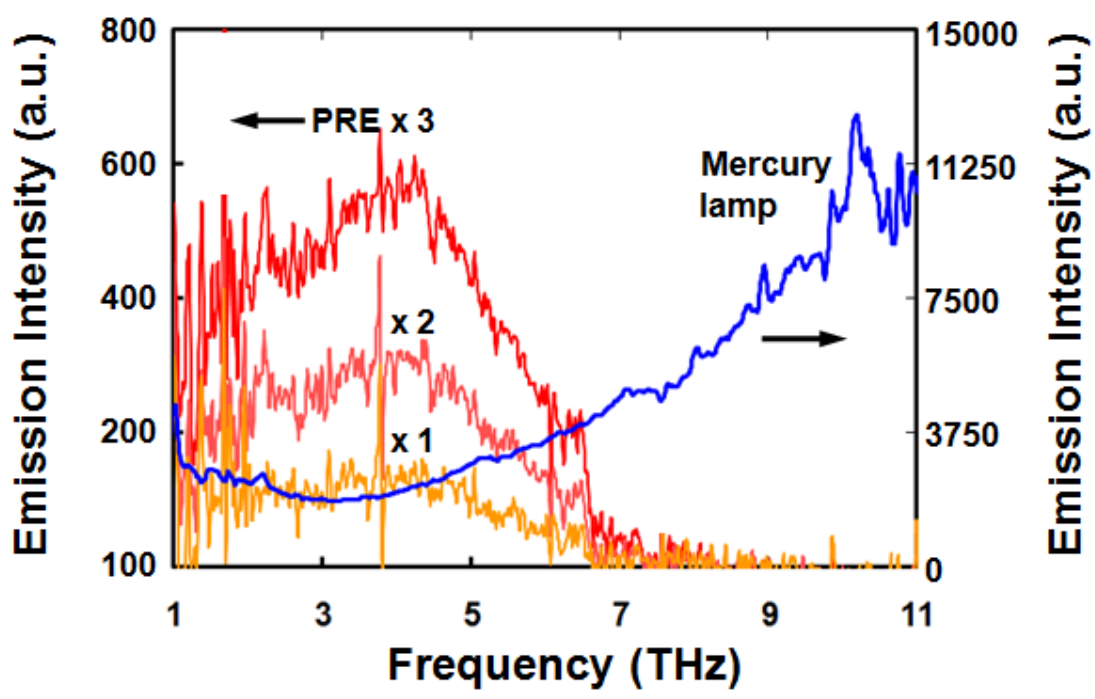


Fig. 5.

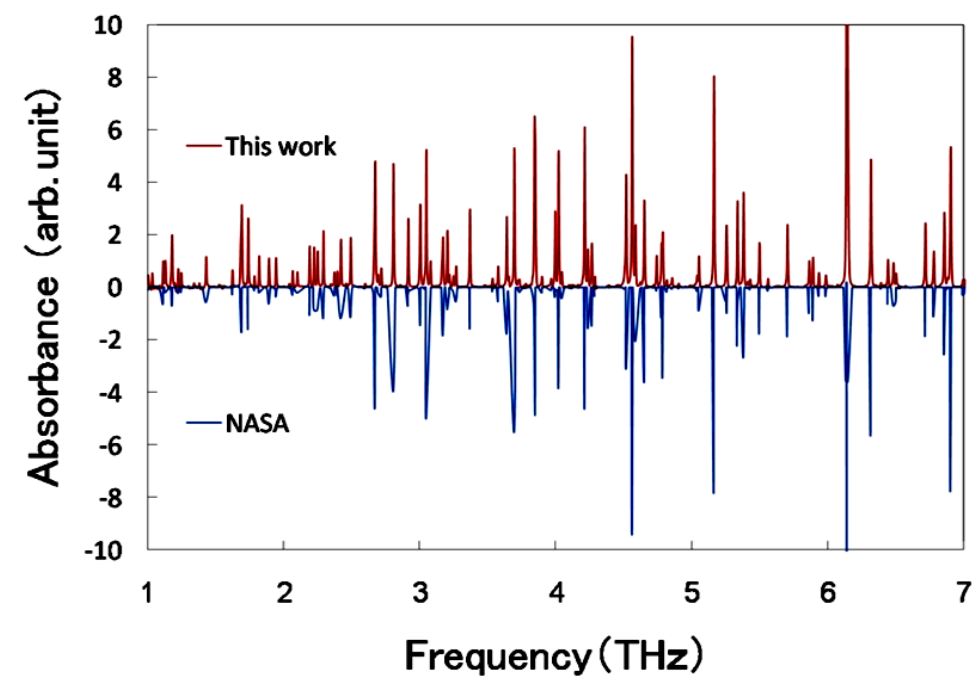


Fig. 6.

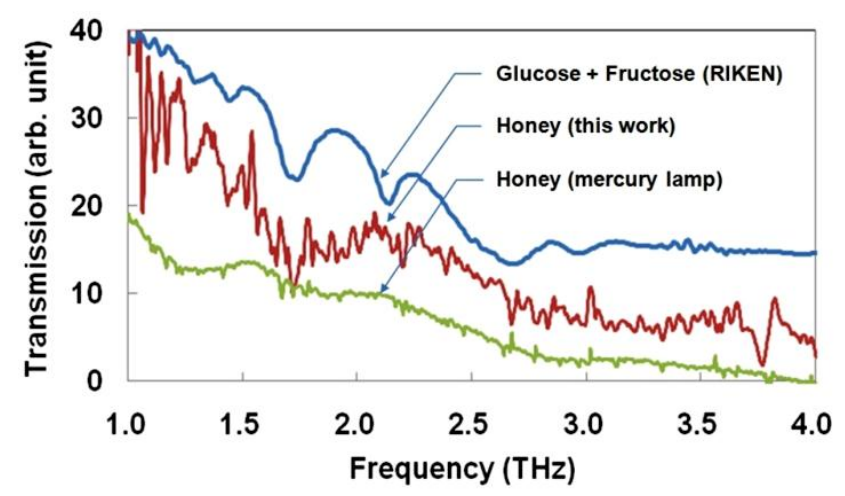

(a)

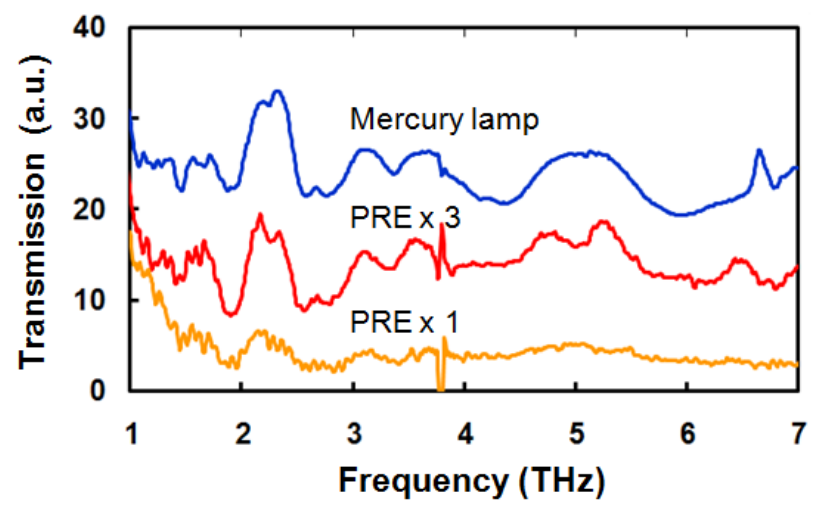

(b) 
Fig. 7.
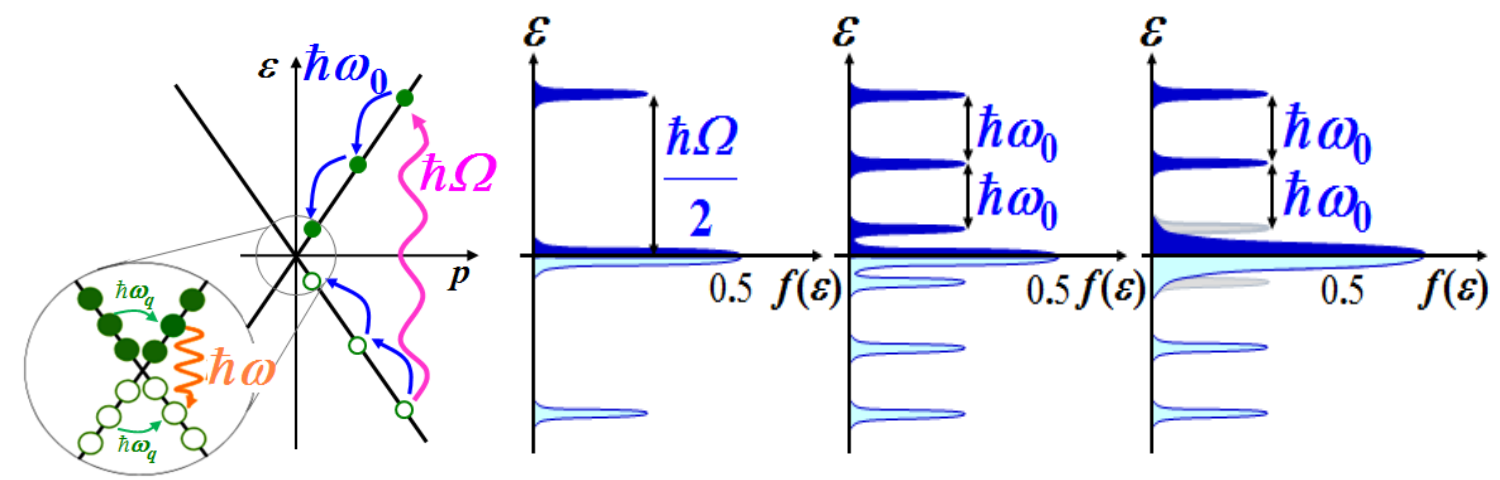

(a)

(b)

(c)

(d) 
Fig. 8.

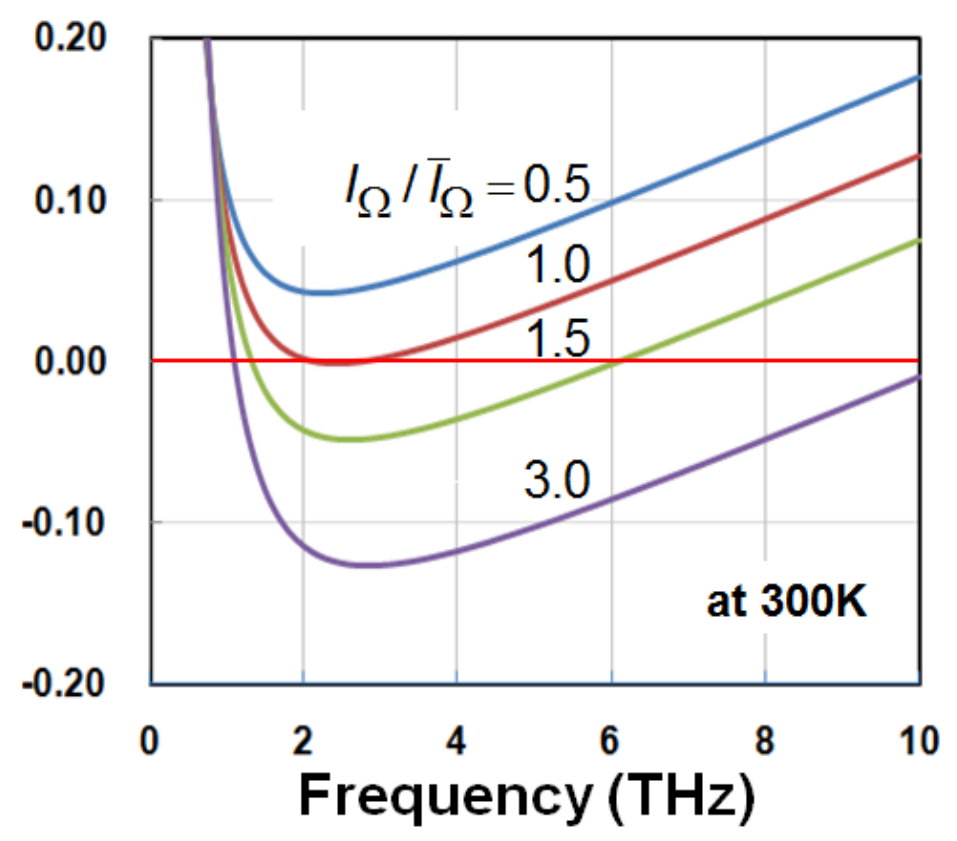


Fig. 9.
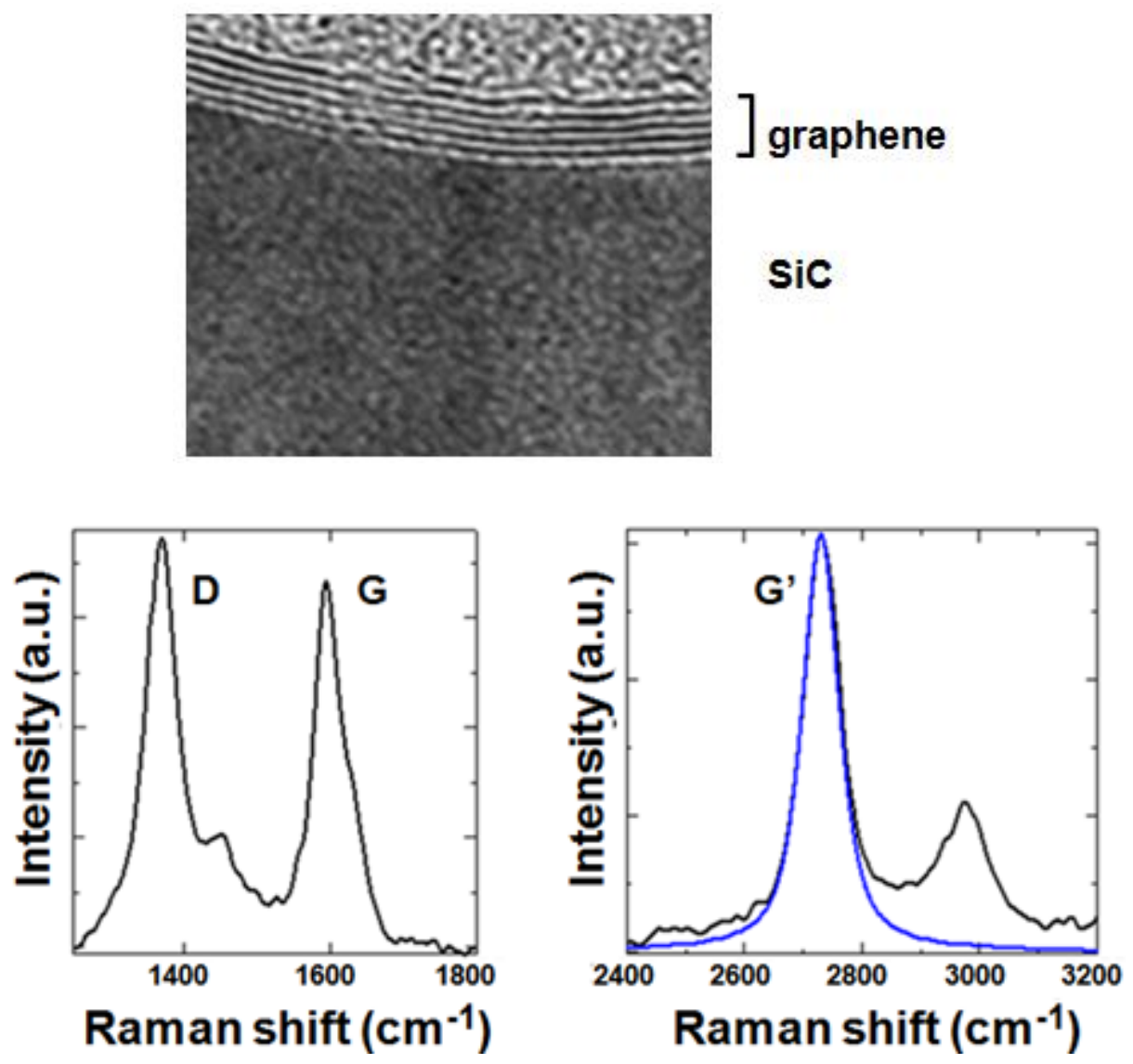
Fig. 10.

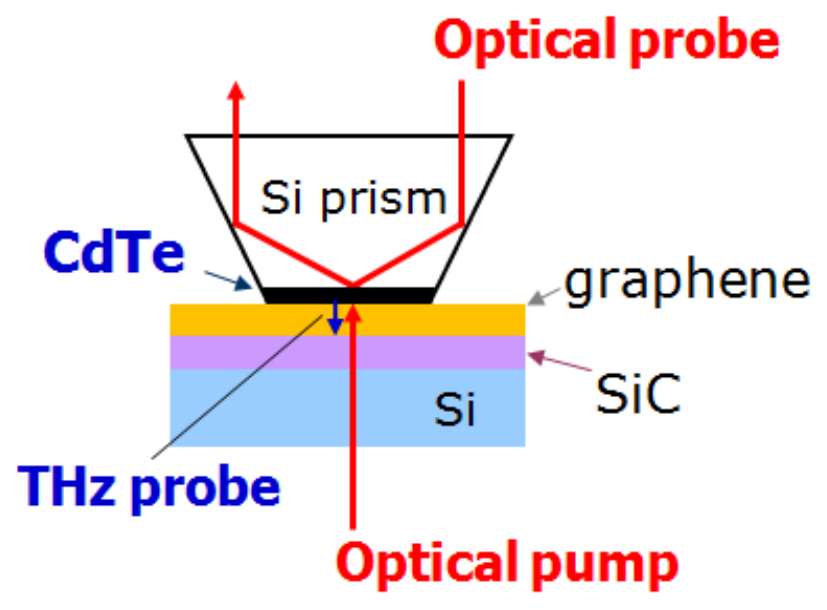


Fig. 11.

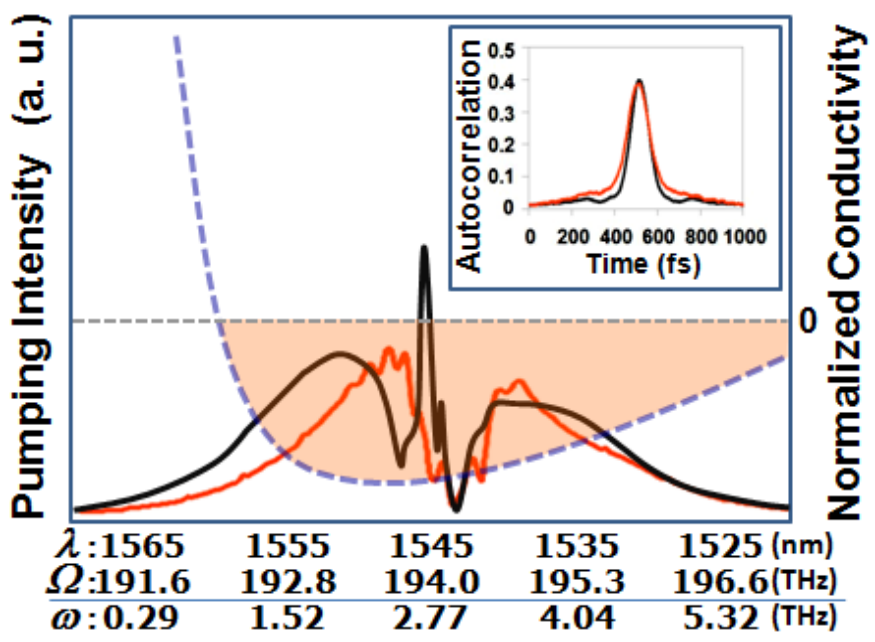


Fig. 12.

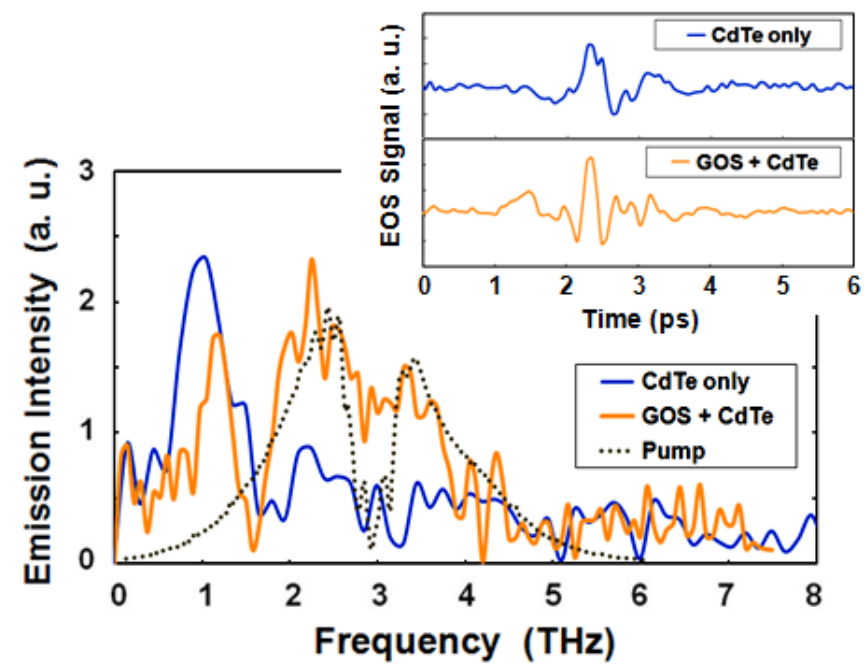

(a)

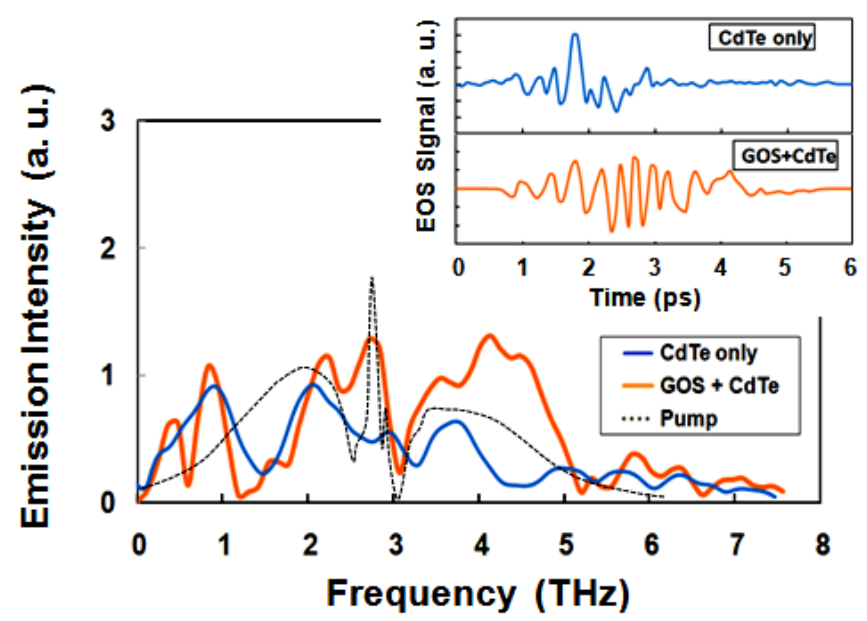

(b) 